\title{
Research on Mechanism and Innovation of Urban Community Sports Construction
}

\author{
Xiuling Zhang ${ }^{1, a}$ \\ ${ }^{1}$ Zheng Zhou Vocational and Technical College; Zheng Zhou; Henan 45000 \\ a email
}

Keywords: urban; community sports construction; institutional choices; mechanism innovation

\begin{abstract}
Urban community sports construction is an important part of the community's cultural and community services. We need to develop urban community sports both sports, but also the community construction and management needs 10 years, Chinese urban community sports building has accumulated some experience, to promote the healthy development of urban community sports development, it is necessary to question the status quo of urban community sports construction rational scrutiny, further adaptation of social transformation and urban community sports construction the reform and innovation.
\end{abstract}

\section{Selection of Urban Community Sports Management System}

Urban Community Sports Management System is led by the party and the government, all sectors of society to participate in regional small community residence resident institutional autonomy management. Physical education through self-residents, self-service sports, sports self-supervision to residents of identity and belonging as a link, forming a co-residence, the common prosperity and common management, build a civilized, peaceful co-insurance social self-management operation mechanism. After Urban Community Sports Construction Practice years, all over the country to form a multi-class management system, its main mode of Shanghai, Shenyang mode, Jianghan mode (Wuhan) and Tianjin mode, the basic construction of community sports practice several cities each distinctive, select the community sports management are not the same. I believe that all regions in the selection of community sports management mode when it is necessary to take into account current conditions, the city of love, from the practical and realistic, but also to see the future trend of urban community sports development, focus on short, medium and long-term urban community contact sports construction, adhere to the unity of the immediate and long-term interests [1].

From the characteristics of cities and the construction of community sports, avoid across the board, windy chaos. In order to take full account of the specific conditions of politics, economy, sports, culture, sports facilities and other urban communities. Administrative color gradually may weaken community sports management. In order to further the reform and transformation of government functions, firmly grasp the guidance and reasonable position to assist, service and supervision. Expansion of urban community sports autonomy as the basic premise and the main content of grassroots democracy, weaken the administrative color urban community sports management from another perspective, that is, to increase democracy color urban community sports management. This was reflected in the expansion of the urban community sports autonomy based urban community grassroots democracy, from a people make decisions by the people to decide. The urban community sports truly become a member of the regional social interests of the community and the regional autonomy of social management support. Given the extent of the existing democratic development of urban community sports, to achieve this objective, we must go through three stages to achieve the two leaps; mainly from government, semi-autonomous administration to half, then gradually move towards self-government.

\section{Innovation of Urban Community Sports Working Mechanism}

Urban Community Sports Construction to achieve the goal, in addition there must be institutional 
innovation as a guarantee, but also need the appropriate mechanism to be implemented. This paper focuses on financing mechanisms and participation mechanisms ${ }^{[2]}$.

Innovative financing mechanism, foreign sources of funding urban community sports activities generally have the following channels: (1) government funding. Daily provision within the community sports city public facilities provided by the government; (2) contributions and taxes. Social fundraising is an important channel for the urban community sports funding sources. Religious organizations and charities will give funding, in Japan, in addition to residents pay income tax, but also to pay a residential area associated with the tax, resident tax. This part of the tax directly for the construction of community sports; (3) self-financing. Financing of the activities in Europe completely autonomous sports organizations sports economically developed countries are generally raised by the organization's members. In addition, leaders of some countries, urban community sports organization is volunteers. At present, the source of funds for construction of urban community sports highly irregular, not yet formed fixed investment. Summed up China's current national conditions, we believe that the construction of urban community sports should adopt multi-channel funds to build social capital investment and government funding combined financing mechanism. Firstly, the Government should increase the construction of urban community sports funding. Mainly for urban infrastructure construction of community sports services, Urban Community Sports Construction of basic work and community sports team-building and community sports organizations office expenses. With the establishment of urban community sport system, governments at all levels should be established for the project community sports organizations, community sports service agencies funded system, and this funding included in the budget at all levels of government.

At present some of our urban community sports Experimental Zone in government funding the positive aspects of try and explore some experimental area will be Community Sports Construction in the annual economic and social development plans, create a budget account in the financial, implementation costs with the matter forward, tax rebates, special financial transfer payment subsidies and so on. Some experimental area by a certain percentage each year new district-level fiscal revenue expenditure additional community sports development, earmarked for the development of community sports undertakings. In order to continue to consolidate these results, continued improvement and development. Second, encourage and attract the community enterprises, institutions, community groups, and individuals to invest in intermediary organizations, the donor community sports development must continue construction of urban community sports service free, low compensation, paid services combine to achieve service support services. To create conditions to improve community sports investment environment and encourage the construction of community sports services to attract businesses to expand revenue sources. To raise funds to mobilize individual residents organize community sports public welfare undertakings, the purpose of co-resident with the community to build the sport. In order to attract more funds for the construction of community sports should develop appropriate policies. Thirdly, the adjustment of tax policy, in order to ensure that the urban community sports building has a relatively stable funding, the proposed introduction of urban community sports development tax.

Furthermore; some small collection of taxes can be entrusted to the community, according to the principles of cost to go along with things, appropriate charges. Such as a bicycle tax, it levied a great difficulty in full if the levy on behalf of the community now, which can solve the difficult bike tax levy issues, but also provide a stable funding for community sports. Fourthly, reform the current new sports lottery soccer lottery, the welfare lottery distribution system. Reasonable distribution by the community, selected ticketing staff, both to solve the employment problem of poor part of the population. In addition, communities can derive certain sales commissions. Siping City, Liaoning Province of soccer lottery, the welfare lottery sales is in this mode of operation, is a successful experience is worth promoting. Fifthly, strengthen the construction of urban community sport from raising funds, use institutionalize management oversight and other aspects, use the funds to carry out strict examination and approval and supervision to ensure the earmarking of funds for construction of urban community sports ${ }^{[3]}$. 


\section{Wide Participation Mechanism Innovation}

Community sports is an important part of community sports, community sports development is inseparable from the context of the development of mass sports. A series of domestic and international studies have shown that mobilizing people to participate in Urban Community sports is an effective way to cultivate people's awareness of community sports. Moreover, the practice of Urban Community Sports Construction that certain city streets in the financial, material relatively strong, the mainly rely on government investment to build a number of community sports facilities in the short term, or to some extent, improve the urban community sports the internal and external environment (such as basic health community sports facilities). However, if no members of the community, especially the active participation of the masses, the government unilaterally work alone, it is difficult to consolidate these construction achievements. It is in this sense that the broad participation of all types of members, especially the residents of the consolidation of the results of Community Sports Construction has important significance.

Community Sports notable feature is its coverage and convenience. Everyone in modern society, he (she) may have no units, no party, but not without a foothold life - community. Community sports organizations, venues and facilities, events, etc., in the community, both to facilitate the organization and management, but also to facilitate residents to participate in these sports may become generalized and living. However, the current situation is the lack of community residents to participate in sports, mainly in two aspects. First, the overall participation rate is not high with uneven distribution. According to the 2003 results of our survey in Beijing, Shanghai, Shenyang, Tianjin, Jinan five cities showed that only 25 percent of residents said gladly participate or willingness to participate in community sports activities or transactions. Uneven distribution, refers to the different age, income, education level of people participation rate is very different. From the age level, the key personnel involved in community sports activities in nearly $70 \%$ of whom are retirees, students accounted for about $10 \%$, only $20 \%$ of young, and if there is no mandatory organization units involved in the proportion of young people will be lower; from income, educational level, the income, educational level, the income, the higher the education level, the lower the community sports participation rate. The second is reflected in the level of participation is not deep. Residents in most cases only participate in sports activities in the community affairs, and rarely participate in decision-making and management ${ }^{[4]}$.

Causes lack of community participation is the cause of many sports, both institutional and individual factors are also conceptual. Units under the unit system formed sense of participation in a large extent hindered the generation of residents to participate in community sports consciousness. After 1949, through ownership units and units under the system of administrative personnel, the government almost all aspects of social welfare, especially in urban social life are included in the unit - under the administrative system. After entering the individual units, which will bear the illness and death of its unlimited obligations, combined with the implementation of the principle of universal distribution of low wages and egalitarian, lack of social services, many individuals also had to rely on institutions to provide benefits (health care, early retirement, housing, education, employment, etc.). Organizational units of the larger society make the pattern between individuals, individuals and social intermediary organizations have contact. This comprehensive personal attachment unit is formed strong personal sense of identity units and units involved in consciousness. With the gradual establishment of China's economic reform, market economy, the units of organization system has undergone great change, some people no longer employed in state-owned units, work units and their relationship has become the main economic relations. Even in state-owned units and units of social security and social welfare workers functions is gradually fade into the same economic relationship based. These organizations, social structure dramatically changes the basic unit of social consciousness and organizations involved, to a certain extent, reduce the dependence on individual units. However, the participation of the old administrative system standard and standard units formed and participatory practices can't be completely removed in the short term, people both in the attitudes or behavior, consciousness and still more accustomed units involved, to participate in community sports culture awareness, it remains to be seen. 
As administration and the community sports management, the residents of the government increase psychological dependence, the concept of autonomy, a sense of participation. Since the Community and street superimposed on geographical zoning, and some even include government officials, a considerable part of the community equivalent to the street that the two different but not fundamentally different name, community sports management but is expanding the scope of street management, content extension and permission to increase. Therefore, the social life, administrative management under long-term planned economy in the construction of community sports still abound, community sports management in fact be defined as an administration, despite the provisions of the Organic Law of the Urban Residents Committees People's Republic of China, neighborhood committee is a resident self-management, self-education, self-service grass-roots mass organizations, the functions of a member elected by the residents to be generated. But in fact, as the organization is autonomous neighborhood street at a government agency sent to become the city's most basic level government administrative bodies, the residents did not participate in the election committees members. This feature results in its neighborhood there is a serious dislocation of the functions in the course, a lot of the other types of organizations and residents should bear the specific transaction. The same old methods of work, the content can't meet the resident individual, personalized, diversified needs, causing residents of the community sports enthusiasm is not high, the use of administrative methods and planning management of community sports, community sports building top-down inevitably some formalism, superficial, pay attention to the momentum, neglect of community sports. Such as the construction of community sports is the most important part - the content of community sports services although rich, but obviously it is mainly the elderly and households, much of the content relates to the individual needs of young people. Community sports activities as well, in order to meet the higher level examination or commemorate a sports festival held in sports and neighborhood streets are often organized activities, one for checks payable, going through the motions, without taking into account residents personalized, diversified demand; the second is the annual content and form are so, can't meet the changing needs of the residents, leading to enthusiastic residents generally not high. Except for some elderly and minority communities interested in sports activities, and the rest were mostly in the organizational unit had to passive participation. The results of several surveys also show that a large number of residents believe that the current organization of community sports activities does not make sense, without the organization units, they do not as a community of active participation.

Improve the living environment and the lack of modern urban venue is not conducive to the objective reality of Community increased sports participation behavior. In large-scale urban construction and urban transformation, relocation and mobility of citizens increased, people familiar with the sport to leave the original living environment, to new and unfamiliar living communities; in the same community, the neighborhood is a low, medium and high-grade points; a high degree of heterogeneity of population, values, lifestyles are very different, this change to the residents cultivate conscious active participation of community sports consciousness objectively created obstacles and difficulties. Residents lack of change in community sports participation, it is imperative, efforts should be two. Make ensure that residents participate in community decision-making construction of sports affairs, through autonomy, cultivate awareness of participation of residents. Residents confirmed the sense of participation from residents of their dominant position in the construction of community sports, and confirm that you want to achieve this in the decision-making process involved in community sports development transaction. In the current residents of autonomy is not strong, self-awareness is not high and the lack of experience of the reality of punishing the government can engage in the vital interests of the residents off, deeply concerned about the residents of affairs start guide residents about the transaction undertaken autonomous participation. Create conditions for residents of multi-channel, multi-form to participate in community sports affairs. Construction of community sports participation is all-round, multi-level, multi-channel, it is not just involved in public service or organized activities and transactions in the current high degree of heterogeneity of population, with the participation of high and low awareness of the situation, the Government may give policy support, to provide the 
necessary activities, so that each community residents from their own needs and characteristics of view, choose different forms of participation and content.

\section{Nonprofit Sports Organization of Urban Community Sports Construction}

Non-profit sports organization, the folk sports service organizations, that is, non-governmental international sports organizations referred to in. Although the non-profit sports organizations in the form of organization of countries, different activities, services, and city community sports development is not as obvious, however, their common features are: Folk autonomy, not profit distribution, voluntary participation, welfare. In developed countries, the urban non-profit sports organizations play a very important role. Its outstanding performance is: to fill the lack of government funding for community sports development. For example, the United States each year about $\$ 50$ billion private investment in the non-profit sports, of which only one individual donor amounted to more than $\$ 10$ billion, greatly complement the government for lack of funds in this regard. Open up a large number of employment opportunities for sports industry. For example, the US non-profit sports organization are about 100000, paid employee are about 100 million people, who is indispensable in the field of employment problem to solve. In addition, volunteers actively involved in non-profit sports organization activities more than 900 million people, formed to promote the development of social sports huge human resources. Increase transparency and the use of sports resources rationality. Due to non-profit sports organizations to run in popular participation and government regulators can better avoid corruption and waste, more fully utilize various social sports resources. It provides a wide range of various types of assistance to vulnerable groups and sports development lags behind urban community sports, thus expanding social equity, alleviate social contradictions, promoting social progress ${ }^{[5]}$.

Chinese urban non-profit sports organizations are still in early stages of growth, but it has shown that it is an important force to promote the urban community sports development. For example, Chinese urban community sports organizations in this social non-profit organization in the last 10 years of hard work to effectively solve the problems of urban vulnerable groups to participate in physical fitness activities in the tens of thousands. In the urban community sports building, the status and role of non-profit sports organizations in the city has become increasingly prominent. More and more cities nonprofit sports organization started by the original management of urban community sport organizations direct government-run, or directly to set up, operate the new urban community sports organization, and has made remarkable achievements. These factors indicate that the urban community non-profit sports organizations are increasingly becoming one of the main forces of urban community sports development.

Therefore, the cultivation and development of urban non-profit sports organizations, and gradually form a good mechanism for these sports organizations to participate in community sports development, and vigorously promote the construction of urban community sports an important part. In this regard, we believe that: the progressive realization of some government agencies, some enterprises and institutions to non-profit sports organizations social restructuring. Foster and develop existing urban community sports organizations play their full role in the urban community sports development process. On the basis of existing urban community mass sports organizations, in accordance with the non-profit community norms and requirements, foster a number of non-profit sports organizations specialized urban community sports construction. Encouraging and supporting urban development and refinement of non-profit sports organizations to actively participate in community sports development policies and regulations, including financial, project, site, tax and other preferential policies, etc., involved in the construction of urban community sports-for-profit sports organizations system utilities provide a good condition.

\section{Conclusions}

Urban community sports construction is an ongoing development of the social movements, but also a social systematic project involving a wide range of fully mobilize social resources, in particular 
the role of the government, community-based organizations (including non-profit sports organizations), community residents three action, so that in the Urban community sports development process do their job, top to bottom with the fundamental guarantee, but Urban community Sports construction coordinated the smooth progress. Therefore, government builds the construction of community sports, community-based organizations, community tripartite cooperation, economic, political, social and cultural three resources to jointly support the city, and its security system is a necessary requirement of the construction of urban community sports.

\section{Acknowledgment}

This is the initial result of Science and Technology Department of Henan Province, 2014 soft science and technology project.

Project Fund Number: 142400410208.

\section{References}

[1] Wangkai Zhen, Ren Hai, Chinese Social Transformation and Urban Social Sports Management System [J] Beijing Sport University, 2004, 27 (4): 433-4399.

[2] Luohan Li Peng Xionghui, Urban Community Sports Condition and Development Strategy [J]. Journal of Physical Education, 2004, 11 (1): 36-37.

[3] Li Jianguo, Development of Community Sports in in Shanghai [J] Shanghai Institute of Physical Education, 2001, 25 (4): 76-78.

[4] Yuanguang Feng, Chen Rong, Chenru Hua, Discussion on Community Sports Resources Development and Utilization [J] Beijing Sport University, 2004, 27 (4): 587-599.

[5] Zhu Hongwei, Theory Analysis of Our Community Sports Construction in Urban [J]. SPORTS AND SCIENCE (Nanjing), 2004, 25 (3): 42-44. 